\title{
't Hooft model on the Lattice
}

\author{
Margarita García Pérez ${ }^{a}$, Antonio González-Arroyo* ${ }^{* a}$, , Liam Keegan ${ }^{c}$ and Masanori \\ Okawa $^{d, e}$ \\ a Instituto de Física Teórica UAM-CSIC, Nicolás Cabrera 13-15, \\ Universidad Autónoma de Madrid, E-28049-Madrid, Spain \\ ${ }^{b}$ Departamento de Física Teórica, C-15 \\ Universidad Autónoma de Madrid, E-28049-Madrid, Spain \\ ${ }^{c}$ Theoretical Physics Department, CERN, 1211 Geneva 23, Switzerland \\ ${ }^{d}$ Graduate School of Science, Hiroshima University, \\ Higashi-Hiroshima, Hiroshima 739-8526, Japan \\ ${ }^{e}$ Core of Research for the Energetic Universe, Hiroshima University, \\ Higashi-Hiroshima, Hiroshima 739-8526, Japan \\ E-mail: margarita.garcia@uam.es, antonio.gonzalez-arroyo@uam.es, \\ liam.keegan@cern.ch, okawa@sci.hiroshima-u.ac.jp
}

Lattice results are presented for the meson spectrum of $1+1$ dimensional gauge theory at large $N$, using the Twisted Eguchi-Kawai model. Comparison is made to the results obtained by 't Hooft in the light cone gauge.

34th annual International Symposium on Lattice Field Theory

24-30 July 2016

University of Southampton, UK

${ }^{*}$ Speaker. 


\section{Introduction}

't Hooft model [1]-[2] is just the 1+1 dimensional version of QCD in the large $N$ limit with quarks in the fundamental representation. Despite the huge expected differences with the $3+1$ dimensional case (no gluons, dimensionful constant, perturbative confinement, no angular momentum, etc) the meson spectrum shows nice resemblances among both cases (chiral limit, Regge behaviour, quark condensate [3], etc). Let us briefly summarize the most salient features. The meson spectrum consists in alternating even and odd parity states, whose masses $\mu_{n}$ asymptotically grow linearly with the integer index $n$ :

$$
\mu_{n}^{2} \sim n \pi^{2}
$$

The meson masses squared are expressed in units of $m_{0}^{2}=g^{2} N / \pi=\lambda / \pi$. Furthermore, the mass squared of the lowest meson state, which we will call pion colloquially, vanishes linearly with the bare quark mass as follows:

$$
\mu_{0}^{2}=\frac{2 \pi}{\sqrt{3}} m_{q}+\mathscr{O}\left(m_{q}^{2}\right)
$$

This mass and those of the excited states can be obtained by solving a 1-dimensional integral equation. Recovering these results using Lattice Gauge Theories provides an interesting challenge. Some results [4] on 1+1 gauge theories have been obtained in the past by standard methods for low values of $N \leq 5$. However, no large $N$ extrapolation has been attempted. The only quantitative study at large $N$ was done by Narayanan and Neuberger [5] using the idea of reduced models [6]. Our approach differs from this one by the use of the twisted reduced model [7]-[8]-[9] instead of the quenched version [10]. We recently [11,12] proposed a method to compute the meson spectrum in $3+1$ dimensions in this context which applies trivially also to the $1+1$ dimensional case. It is certainly a good testing ground for our methodology since the meson masses in the continuum are known from Ref. [1].

\section{Methodology}

In this section we are going to briefly describe the main aspects of our method. As shown in Ref. [6] the expectation value of Wilson loops in the large $\mathrm{N}$ limit becomes independent of the space-time volume. Hence it is possible to evaluate these loops by simulating a lattice model with a single point. This can be done either with the original periodic Eguchi-Kawai prescription (EK), the quenched version (QEK) [10] or the twisted version (TEK) [7]-[8]. In 2D the conditions necessary for a proof of reduction are met in all cases. Nevertheless, large $N$ corrections are much larger for EK than for QEK and TEK. Paradoxically the calculation of rectangular Wilson loop expectation values at large $N$ for the infinite volume setting is simpler and follows an exact area law [13] with a string tension given by $-\log (1-1 /(4 b))$ where $b$ is the lattice equivalent of the inverse ' $t$ Hooft coupling $\lambda$ corresponding to Wilson action.

In this work we will be using the twisted version of the matrix model whose partition function is given by

$$
Z=\int d U_{0} d U_{1} \exp \left\{b N z \operatorname{Tr}\left(U_{0} U_{1} U_{0}^{\dagger} U_{1}^{\dagger}+\text { h.c. }\right)\right\}
$$

where $b$ is the afore-mentioned lattice coupling $\left(b=1 / \lambda_{L}\right)$, the matrices $U_{\mu}$ belong to $\mathrm{SU}(N)$ and $z=e^{2 \pi i k / N}$ is an $N$-th root of unity ( $k$ is chosen co-prime with $\left.N\right)$. 


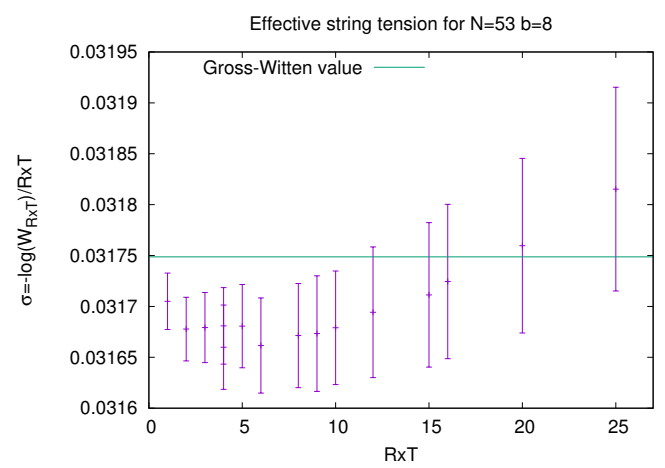

Figure 1: Logarithm of the expectation value of the Wilson loop divided by the area.

In this theory rectangular Wilson loops can be constructed in terms of the two matrices $U_{0}$ and $U_{1}$ as follows:

$$
W_{R \times T}=\frac{z^{R T}}{N}\left\langle\operatorname{Tr}\left(U_{0}^{T} U_{1}^{R} U_{0}^{-T} U_{1}^{-R}\right)\right\rangle
$$

Volume reduction implies that in the large $N$ limit these expectation values would coincide with those obtained for standard Wilson loops in the infinite volume theory. Indeed, this can be tested. For example, in Fig 1 minus the logarithm of the expectation value divided by the area of the loop is plotted as a function of area for several Wilson loops at $b=8$ and $N=53$. Agreement with the exact area law and string tension evaluation is obtained to within a few permille level.

An important question when using the reduced model is what is the size and nature of finite $N$ corrections. The perturbative analysis [8] reveals that part of the $1 / N$ corrections has the same form as that of a finite volume correction for a lattice volume of size $N^{2}$. This idea is very helpful in determining the values of $N$ that one must choose to minimize the effect of finite $N$ errors. The conclusion is that the correlation lengths should be kept much smaller than the effective length scale of our box, which is $N$.

Our main goal is to obtain the meson spectrum for this model. We will follow the same procedure that we employed for the 4 dimensional case [11]. The idea is that, given that gauge fields are effectively living in an $N \times N$ box, the same can be chosen for the quark fields. Indeed, one can duplicate the size in the time direction to avoid the need of using cosine hyperbolic instead of simple exponentials when fitting the time dependence of correlators. Then, the analysis performed in Ref. [11] implies that the meson correlators at zero spatial momentum are given by

$$
C_{A B}(t)=\sum_{p_{0}} e^{i p_{0} t}\left\langle\operatorname{Tr}\left(O_{A} D^{-1}\left(p_{0}\right) O_{B} D^{-1}(0)\right)\right\rangle
$$

where $O_{A}$ and $O_{B}$ are operators defined at fixed time and are meant to project onto meson states of the right quantum numbers. In our case the only quantum number is parity. The symbol $D^{-1}\left(p_{0}\right)$ stands for the lattice quark propagator where the time dependence has been Fourier transformed. In principle one is free to choose any possible version of lattice fermions. In this work we are using Wilson fermions, naive fermions and overlap fermions. As a matter of fact, we can use our simple system to test the possible advantages and disadvantages of each choice. The spectra can be different at fixed lattice spacing, but should agree with ' $t$ Hooft values in the continuum limit. 
Here we will show that nice results can be obtained for all cases, but we defer a full comparison for a future publication.

In any case, the propagator is obtained by inverting the corresponding Dirac operator which is a $2 N^{2} \times 2 N^{2}$ matrix. The covariant derivative appearing in the operator has to be replaced by

$$
D_{\mu} \longrightarrow U_{\mu} \otimes \tilde{\Gamma}_{\mu}-\mathbf{I}
$$

where $U_{\mu}$ are the gauge matrices of the reduced model and the $N \times N$ matrices $\tilde{\Gamma}_{\mu}$ act on spatial coordinates. Its actual form is irrelevant because it is easy to see that they satisfy

$$
\tilde{\Gamma}_{0} \tilde{\Gamma}_{1}=z \tilde{\Gamma}_{1} \tilde{\Gamma}_{0}
$$

and this equation has a unique solution having $\tilde{\Gamma}_{\mu}^{N}=\mathbf{I}$ modulo similarity transformations. The latter are irrelevant since we are projecting over zero spatial momentum.

Concerning the operators $O_{A}$ employed in our analysis we have followed an analogous implementation to the one we used for the four-dimensional case, reported by M. Okawa in this conference [14]. One could choose ultralocal operators, in which case they act only on the spinor indices. The unit matrix and the three Pauli matrices form a complete set. To reduce the contribution of the excited states one can use operators having a spatial extent. This is done by applying a smearing operator $D_{s}$ which adds a contribution one lattice spacing apart. This can be iterated several times to give operators with different smearing levels which can then be used in a variational determination of the energy eigenstates. For details we refer the reader to our future publication.

We also make a few comments about the numerical aspects of our work. We have analysed data for various values of $N: 31,43$ and 53 and various values of $b$ in the range [3,8]. In each case we generated 1000 gauge field configurations using the over-relaxation method of Ref. [15]. This step takes minutes in a standard PC for these values of $N$. The matrix inversions are performed with the source method. The procedure can be carried on a PC or a small cluster. The total time employed depends on the number of smearing levels considered.

As mentioned earlier in our analysis we use several different lattice operators. As in our four dimensional studies we use Wilson fermions. This has two major disadvantages. One is the existence of an additive quark mass renormalization which forces to make a fit in the hopping parameter to determine its critical value corresponding to zero bare quark mass. On the other hand one expects lattice artifacts of order $a$ rather than $a^{2}$. Our second option is to take naive fermions. Both of the mentioned problems are solved at the expense of generating doubling. However, in our case there are no dynamical fermions and the doubling only affects valence quarks. This generates some inessential complications in extracting the meson masses. Finally, we also use massive overlap fermions, with the conventions of Ref. [16]:

$$
D_{\mathrm{OV}}=\frac{1}{2}\left(1+\mu+(1-\mu) \gamma_{5} \operatorname{sign}\left(\gamma_{5} D_{\mathrm{WD}}(-m)\right)\right)
$$

where $D_{\mathrm{WD}}(-m)$ is the Wilson Dirac operator with negative mass $m$. The main disadvantage is that this takes longer to invert. However, using several optimizations and the multishift conjugate gradient method to invert several masses simultaneously, the total time cost of using the overlap is only a factor 10 to 15 bigger than that for Wilson fermions. 


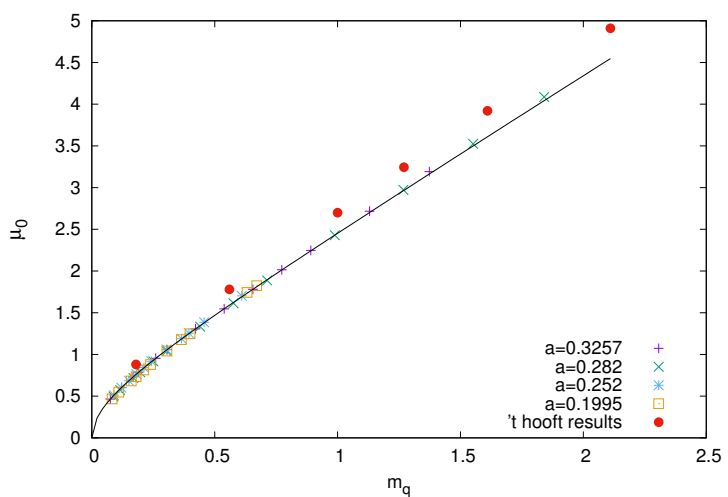

Figure 2: Lowest meson mass for Wilson fermions

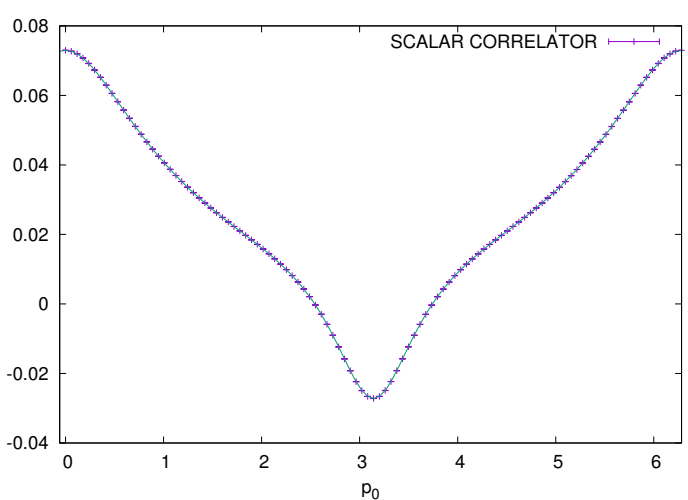

Figure 3: Correlator in momentum space for naive fermions $N=53$ and $b=8$

The final comment relates to the methodology used to extract the masses. The most naive methodology would be perform a multi-exponential fit to the correlation function in time. A better alternative is to use a variational analysis similar to the one employed in four dimensions. The systematic errors of these methods arise from the choice of range in which to perform the fits. This can be avoided if one extracts the masses from a fit to the correlation function as a function of $p_{0}$ as done in Ref. [5]. This, however, has other types of systematic errors emerging from the parameterization of this correlator. In any case, the final results of our study should show the relative benefits of the different methods.

\section{Results}

In this section we will present some of the preliminary results of our study, separating the analysis between the different types of Dirac operators.

\subsection{Wilson fermions}

The procedure in this case is similar to the one that we have followed in the four dimensional case. We generated 1000 configurations at $N=31$ and various values of $b(3,4,5$ and 8$)$, which correspond to a lattice spacing of $a=1 / \sqrt{\pi b}$ expressed in the mass unit mentioned in the introduction. Then we computed the meson correlator for various values of the hopping parameter $\kappa$. This is converted to a lattice bare quark mass given by the formula

$$
M_{q}=a m_{q}=\log \left(1+\frac{1}{2 \kappa}-\frac{1}{2 \kappa_{c}}\right)
$$

The value of $\kappa_{c}$ is obtained from a fit of the lowest meson mass square to a linear plus quadratic function of $M_{q}$. Our results for this meson mass are displayed in Fig. 2 as a function of $m_{q}$. They seem to scale quite well, but the values are smaller than the corresponding values of Ref. [1]. At this stage it is hard to know what is the origin of the discrepancy. It could be due to finite $N$ corrections, but most probably to a mismatch between the definition of quark mass on the lattice and the equivalent in ' $t$ Hooft paper. Indeed, a rescaling of the lattice quark mass by a factor 0.9 makes the data fall on top of " $t$ Hooft data points. 


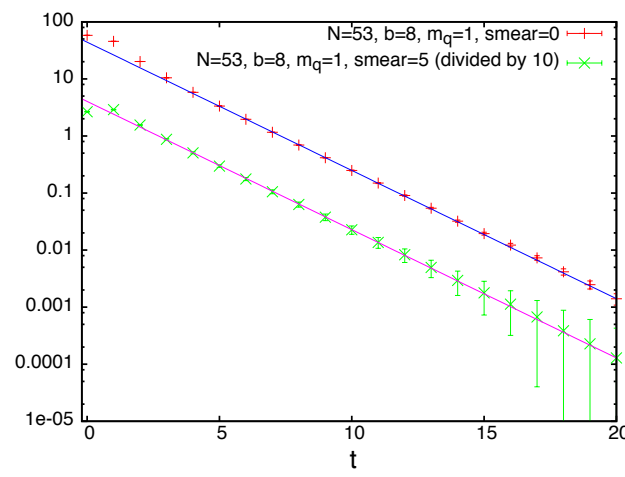

Figure 4: Correlators in position space for overlap fermions.

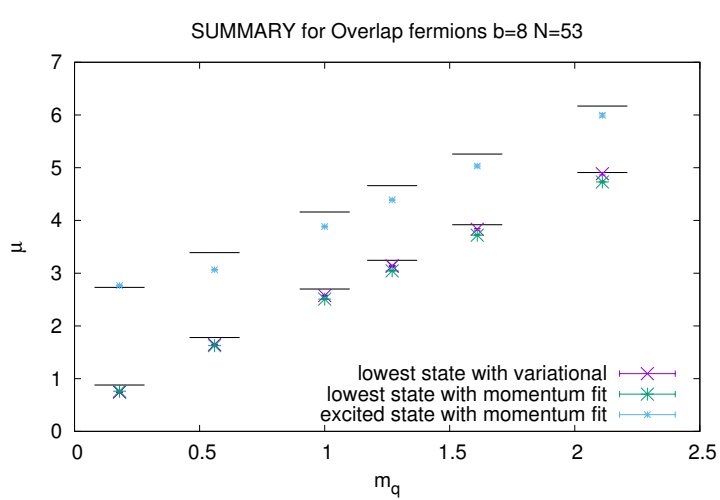

Figure 5: Summary of meson masses for various quark masses for overlap fermions.

\subsection{Naive fermions}

The necessity to fit a value of critical $\kappa$ can be eliminated if we study naive fermions, a special projection of which gives rise to staggered fermions. As a counterpart one has to deal with the phenomenon of doubling. However, in this case this problem can be easily handled. On one side there will be a double quasi-degeneracy of all states. The degeneracy is only exact if $N$ is even. This doubling of states demands a similar enlargement of the space of operators in a variational analysis. In addition, there is a symmetry of the quark propagator as follows:

$$
D^{-1}\left(p_{0}\right) \longrightarrow \gamma_{1} D^{-1}\left(p_{0}+\pi\right) \gamma_{1}
$$

This implies that the meson propagator in $p_{0}$ space contains contributions of both parity even and odd states. For example, for the $\bar{\psi} \psi$ case we can then parameterize its correlator as follows

$$
C\left(p_{0}\right)=\sum_{i=0}^{M} \frac{c_{2 i+1}}{\sin ^{2}\left(p_{0} / 2\right)+\sinh ^{2}\left(\mu_{2 i+1} a / 2\right)}-\sum_{j=0}^{M^{\prime}} \frac{c_{2 j}}{\cos ^{2}\left(p_{0} / 2\right)+\sinh ^{2}\left(\mu_{2 j} a / 2\right)}
$$

Very massive states give a constant contribution and only a few individual masses can be extracted. Fig. 3 shows that a very good description of the data $\left(\chi^{2} /\right.$ ndof $\left.=0.47\right)$ can be obtained using this parameterization with $M=M^{\prime}=1$ (4 meson states) and a constant term mimicking the more massive states. A simultaneous fit to all correlators and operators can be done in order to extract the lowest lying masses. This can be done for all lattice fermion cases.

\subsection{Overlap fermions}

To conclude we give a brief snapshot of our results with the overlap operator. In Fig. 4 we show the correlator in time for the $\gamma_{5}=\gamma_{1} \gamma_{2}$ channel with and without smearing. The exponential fall-off is obvious after the first few values of $t$. Smearing improves the agreement for smaller values of $t$ but the errors become larger for higher $t$. In any case the two operators give compatible values for the mass.

To conclude this presentation we display in Fig. 5 a summary of our present results. The two lowest-lying states are shown at various values of the quark masses compared with the corresponding values as given by ' $t$ Hooft shown as horizontal lines in the figure. The qualitative agreement is 
quite clear, but the lattice values tend to be slightly smaller. In any case a full analysis of systematic errors is still missing.

\section{Conclusions}

In this paper we have presented preliminary results of our analysis of the meson spectra of 2 dimensional QCD in the large $N$ limit. Our results are qualitatively in agreement with the continuum results of Ref. [1]. For a quantitative comparison an appropriate connection of the quark mass in the continuum and on the lattice is necessary. The full results will be presented in a future publication. Our study serves as a testing ground for calculations of the meson spectra for other theories with fermions in the adjoint or two-index representation where the same techniques can be applied in the analysis of results.

\section{Acknowledgments}

We acknowledge financial support from the grants FPA2012-31686, FPA2012-31880, FPA201568541-P, and the Spanish MINECO's "Centro de Excelencia Severo Ochoa" Programme under grant SEV-2012-0249. M. O. is supported by the Japanese MEXT grant No 26400249 and the MEXT program for promoting the enhancement of research universities. Computations have been done with the clusters and PC's at IFT.

\section{References}

[1] G. 't Hooft, Nucl. Phys. B 75 (1974) 461.

[2] G. 't Hooft, Proceedings of the 13th International School of Subnuclear Physics: New Phenomena in Subnuclear Physics; Erice Subnucl.Phys.1975:0261.

[3] A. R. Zhitnitsky, Phys. Lett. 165B (1985) 405 [Sov. J. Nucl. Phys. 43 (1986) 999] [Yad. Fiz. 43 (1986) 1553].

[4] F. Berruto, L. Giusti, C. Hoelbling and C. Rebbi, Phys. Rev. D 65 (2002) 094516 [hep-lat/0201010].

[5] J. Kiskis, R. Narayanan and H. Neuberger, Phys. Rev. D 66 (2002) 025019 [hep-lat/0203005].

[6] T. Eguchi and H. Kawai, Phys. Rev. Lett. 48 (1982) 1063. doi:10.1103/PhysRevLett.48.1063

[7] A. Gonzalez-Arroyo and M. Okawa, Phys. Lett. B 120 (1983) 174.

[8] A. Gonzalez-Arroyo and M. Okawa, Phys. Rev. D 27, 2397 (1983).

[9] A. Gonzalez-Arroyo and M. Okawa, JHEP 07 (2010) 043 [arXiv:1005.1981 [hep-th]].

[10] G. Bhanot, U. M. Heller and H. Neuberger, Phys. Lett. 113B (1982) 47.

[11] A. Gonzalez-Arroyo and M. Okawa, Phys.Lett. B755 (2016) 132, [arXiv:1510.05428 [hep-lat]].

[12] A. Gonzalez-Arroyo and M. Okawa, PoS LATTICE2015 (2016) 291 [arXiv:1511.00477 [hep-lat]].

[13] D. J. Gross and E. Witten, Phys. Rev. D 21 (1980) 446.

[14] M. García Pérez, A. González-Arroyo, and M. Okawa, PoS LATTICE2016 (2016) 329.

[15] M. García Pérez, A. González-Arroyo, L. Keegan, M. Okawa and A. Ramos, JHEP 06 (2015) 093 [arXiv:1505.05784 [hep-lat]].

[16] R. G. Edwards, U. M. Heller and R. Narayanan, Phys. Rev. D 59 (1999) 094510 [hep-lat/9811030]. 